\title{
Designing large-bandwidth planar photonic crystal waveguides
}

\author{
Lavrinenko, Andrei; Søndergaard, Thomas
}

Published in:

Summaries of Papers Presented at the Lasers and Electro-Optics, 2002. CLEO '02. Technical Digest.

Link to article, DOI:

10.1109/CLEO.2002.1033372

Publication date:

2002

Document Version

Publisher's PDF, also known as Version of record

Link back to DTU Orbit

Citation $(A P A)$ :

Lavrinenko, A., \& Søndergaard, T. (2002). Designing large-bandwidth planar photonic crystal waveguides. In Summaries of Papers Presented at the Lasers and Electro-Optics, 2002. CLEO '02. Technical Digest. (Vol. 1, pp. 15-16). IEEE. https://doi.org/10.1109/CLEO.2002.1033372

\section{General rights}

Copyright and moral rights for the publications made accessible in the public portal are retained by the authors and/or other copyright owners and it is a condition of accessing publications that users recognise and abide by the legal requirements associated with these rights.

- Users may download and print one copy of any publication from the public portal for the purpose of private study or research.

- You may not further distribute the material or use it for any profit-making activity or commercial gain

- You may freely distribute the URL identifying the publication in the public portal 


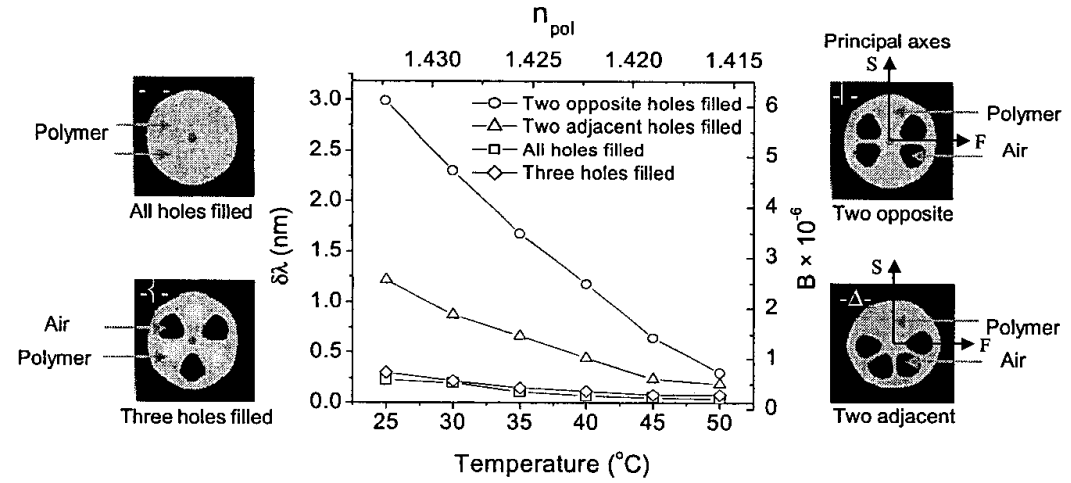

CMC2 Fig. 3. (a) Wavelength splitting of the resonance $(\delta \lambda)$ as a function of temperature for all airholes filled $(--)$, two adjacent airholes $(-\Delta-)$, three alternate airholes $(-\{-)$, and opposite airholes $(-\mid-)$ filled with index tunable polymer.

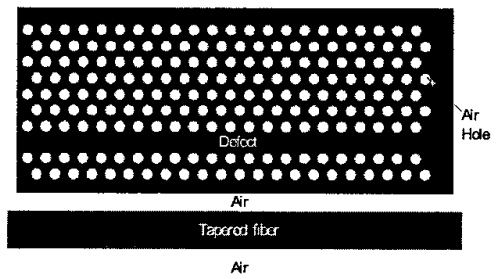

CMC3 Fig. 1. Schematic of the fiber and photonic crystal waveguide coupling scheme.

1 micron so that the propagation field stretches outside the fiber cladding. The refractive index of the fiber is taken to be 1.46 in the simulation. The separation of the tapered fiber and dielectric slab is 0.5 micron. The photonic crystal waveguide is formed by a two-dimensional triangular lattice of air holes in a dielectric slab. The photonic crystal waveguide slab is simulated in two dimensions using an effective refractive index, $n_{\text {eff }}$ of 2.76 . This has been demonstrated to be a good approximation to the three dimensional calculation. ${ }^{3}$ We choose the filling factor of air holes to be $\sim 0.3$ or radii of air holes to be $0.29 a$. The lattice has a TE photonic bandgap from $a / \lambda_{0}=0.256$ to $0.320 .^{3}$ Two rows of air holes on the edge of the dielectric function as a grating and provide field confinement for the photonic crystal waveguide. These rows afford the mechanism for contra-directional coupling under the phase matching condition,

$$
k_{f i b e r}+k_{p c}=\frac{2 \pi}{a}
$$

Here, $k_{f i b e r}$ and $k_{p c}$ are the equivalent propagation constants in the fiber and the photonic crystal waveguide, respectively. $a$ is the lattice constant of the photonic crystal.

Optical structures with 120 photonic crystal periods in the propagation direction are modeled. This domain is significantly longer than that

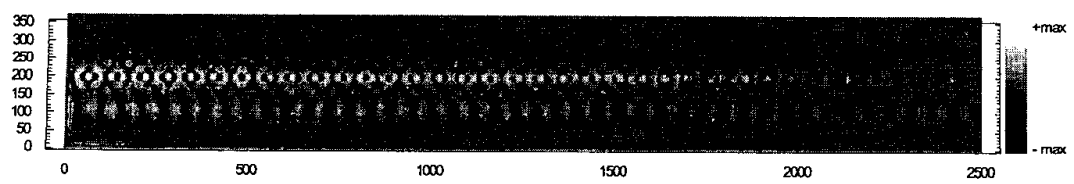

CMC3 Fig. 2. Magnetic field magnitude profile showing contra directional coupling of energy from fiber to a photonic crystal waveguide (2-D FDTD simulation).

hown in figure 1. Discretization is 20 points per interhole spacing. The computational domain, which consists of $2500 \times 350$ square cells, is terminated by Berenger's PML-ABC boundary condition. The field is excited continuously in the fiber with an electric dipole with frequency centered at $a / \lambda_{0}=0.2715$, and the source is positioned at 50 points away from the left boundary in the fiber. Figure 2 shows the coupling along the photonic crystal structures. The power flowing from left to right inside tapered fiber gets significantly weaker, while the power flowing from right to left in the photonic crystal waveguide becomes stronger with the interaction.

It is shown in figure 3 that the coupling efficiency of the proposed approach improves with increasing interaction length between the fiber and the photonic crystal waveguide. Theoretically, all of the power flowing in the fiber can be coupled into the photonic crystal waveguide with an infinitely long interaction length. Practically, a 60 -micron interaction (which is approximately 2400 points in Figure 3) gives a coupling efficiency above $90 \%$ at a 1.55 -micron wavelength This is significant because this length is well

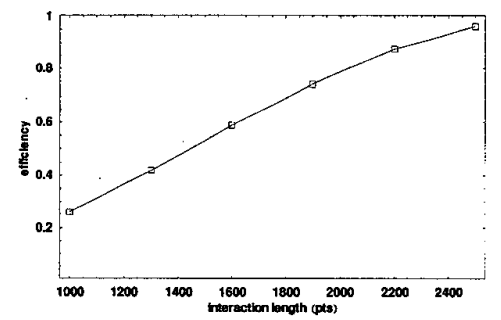

CMC3 Fig. 3. Coupling efficiency as a function of fiber-waveguide interaction length, showing that the method is capable of $90 \%$ and higher coupling. suited for integrated optics. Due to the symmetry of the structure, the coupling scheme works for both input coupling and output coupling.

In summary, this coupling method, which makes use of currently available manufacturing processes, results in over $90 \%$ coupling efficiency.

1. E. Yablonovitch, "Inhibited spontaneous emission in solid-state physics and electronics", Phys. Rev. Lett. 58, no. 20, 2059-2062 (1987).

2. J.D. Joannopoulos, R.D. Meade, J.N. Winn, Photonic crystals-Molding the flow of light (Princeton Univ. Press, 1995), Chap. 7.

3. A. Chutinan, S. Noda, "Waveguides and waveguide bends in two-dimensional photonic crystal slabs", Phys. Rev. B, 62, no. 7 , 4488-4492 (2000).

CMc4

9:00 am

\section{Designing Large-Bandwidth Planar Photonic Crystal Waveguides}

Andrei Lavrinenko and Thomas Sondergaard, Research Center COM, Technical University of Denmark, Building 345v, DK-2800 Kgs.Lyngby, Denmark,Email: ala@com.dtu.dk

The research on planar photonic crystal waveguides (PCW's) is presently to a large extent focusing on PCW designs where the effective refractive index of the waveguiding region is high compared to that of the surrounding photonic crystal. From calculated dispersion relations it has been found that the guided electromagnetic modes in this type of waveguide are often characterized by a very low group velocity, and guidance is achieved only for rather limited frequency intervals (see e.g. [1]). The purpose of this paper is to provide design principles that will make possible the realization of planar PCW's that support leakage-free bandgap guidance of light over large frequency intervals.

Our design idea is best illustrated by giving an example. In Fig. 1 we consider the dispersion properties for a PCW based on introducing a line defect in a photonic crystal with air-holes arranged periodically on a triangular lattice in silicon (dielectric constant 12). The diameter of the air-holes is $D=0.66 \Lambda$, where $\Lambda$ is the lattice constant. The waveguide is shown as an inset. The di-

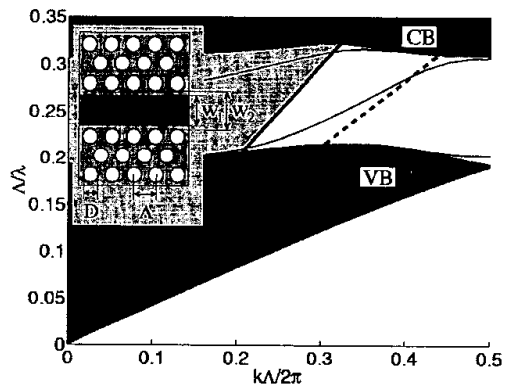

CMC4 Fig. 1. Banddiagram for a photonic crystal waveguide based on introducing a line defect in a photonic crystal where air-holes are arranged on a triangular lattice in silicon. The line defect consists of a silica region with refractive index $\mathrm{n}=1.4$ and width $1.4 \Lambda$. The inset shows the structure. 
agram shows the allowed combinations of Bloch wave vector $k$ and normalized frequency $\Lambda / \lambda$, where $\lambda$ is the free space wavelength. The continuums $V B$ and $C B$ correspond to modes of the photonic crystal material surrounding the waveguide. Between $V B$ and $C B$ there is a photonic bandgap. The dashed line shows the dispersion curve for bulk silica (dielectric constant 2.0), and silica was chosen because the dispersion curve of bulk silica runs through the entire photonic bandgap of the photonic crystal. We can expect that if we introduce a wide line defect in the photonic crystal consisting of silica as shown in the inset there will be guided modes that to some extent follow the dispersion curve of bulk silica. The discrete bands (thin solid curves) correspond to the guided modes for the case of a waveguide width given by $W_{1}=1.4 \Lambda$ and $W_{2}=W_{1}+\Lambda-D$. The thick solid line indicates the light line. Leakage-free guidance of light by the waveguide is only possible for the mode solutions that correspond to discrete bands below the light line for a finite-height PCW suspended in air. ${ }^{2}$ In Fig. 1 there is one discrete band below the light line that follows the dashed curve. Our waveguide design is therefore characterized by first of all a large bandwidth, and secondly it is characterized by a relatively high group velocity giving a better modal dispersion match with the modes of standard waveguides used for coupling light into the PCW.

The example given above demonstrates the general idea for obtaining a large bandwidth. The general idea is not, however, restricted to line defects consisting of a glass channel. A similar effect can be obtained by using e.g. a variety of microstructured materials in the line defect when the dispersion relation of those materials runs through the photonic bandgap. A two- and threedimensional plane-wave-expansion analysis of a variety of large-bandwidth PCW's will be presented at the conference.

\section{References}

1. E. Chow, S. Lin, J. Wendt, S. Johnson, and J. Joannopoulos. Quantitative analysis of bending efficiency in photonic-crystal waveguide bends at $\lambda=1.55 \mathrm{~mm}$ wavelengths, Opt. Lett. 26 (2001) 286-8

2. T. Søndergaard, A. Bjarklev, J. Arentoft, $M$. Kristensen, J. Erland, J. Broeng, S.E. Barkou Libori. Designing finite-height photonic crystal waveguides: confinement of light and dispersion relations, Opt. Comm. 194 (2001) 341-51.

\section{CMC5}

9:15 am

Rigorous Modal Solution of a Multi Mode PCF Using Scattering Matrix Method

Waleed S. Mohammed, Eric G. Johnson and Laurent Vaissé, CREOL/School of Optics,

University of Central Florida, Orlando, Fl

32816-2700, Email: wsoliman@creolmail.org

Photonic crystal fibers (PCF's) have recently gained a great deal of interest for their remarkable waveguiding properties. ${ }^{1}$ This new kind of fiber relies on the photonic band created by a deformed $2 \mathrm{D}$ periodic pattern of air rods in dielectric medium to guide the light. Moreover, control over the hole pattern enables tailoring modal and dispersion properties without having to resort to complex fiber perform.

Waveguiding properties of PCFs have been investigated using plane wave expansion methods. ${ }^{3,4}$ However, $\mathrm{Ez}$ and $\mathrm{Hz}$ coupling is neglected in this formulation. in this paper we utilize a full vectorial formulation for the wave equation as applied to PCF's. This rigorous approach handles any cavity shape or distribution of holes for exact calculations of the complex coupled modes.

The inhomogeneous problem in PCF is divided in two homogeneous problems. The fields are derived inside and outside the rods, taking into account the effect of the structure. The scattered light from each rod is calculated from Maxwell's equations with the appropriate application of the boundary conditions at that rod. Multiple scattering effects on each rod from the surrounding rods are included in the continuity conditions through a so-called local incident light. Finally, the total scattered field at all points in space is given by the superposition of the field scattered from each individual rod.

The $\mathrm{z}$ components of the propagating modes

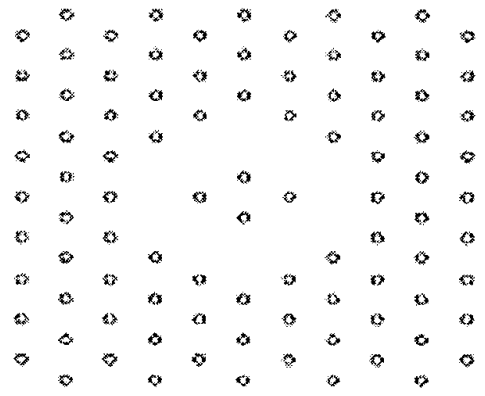

CMC5 Fig. 1. Ring shaped cavity. of the Electric and Magnetic Fields are represented in cylindrical coordinates with ${ }^{5}$

$$
\bar{F}(r, \phi, z)=\bar{F}(r, \phi) e^{i(\varpi t-\beta z)}
$$

$\beta$ corresponds to the $z$ component of the wave vector. The field components at each rod are represented by their Hankel/Bessel expansion as presented in. ${ }^{6}$ Applying the continuity of the tangential field components, $z$ and $\phi$, at the air rod interface, we get the following two vector equations.

$$
\begin{gathered}
\overline{\bar{A}}_{1} \cdot \bar{a}+\overline{\bar{B}}_{1} \cdot \bar{b}=\bar{c} \\
\overline{\bar{A}_{2}} \cdot \bar{a}+\overline{\bar{B}}_{2} \cdot \bar{b}=\overline{\bar{C}}_{2} \cdot \bar{c}
\end{gathered}
$$

Vectors $a, b$ and $c$ represent the expansion coefficients of the $z$ components of the electric and magnetic fields of the local incident, scattered and transmitted light respectively. $\bar{A}_{1}, \bar{A}_{2}, \bar{B}_{1}, \bar{B}_{2}$ and $\bar{C}_{2}$ are square matrixes result from the boundary conditions. From (2) and ( 3 ) and using the linear relation between $a$ and $b$ derived from the local incident field, we get the following relation

$$
\overline{\bar{M}}(\beta) \cdot \bar{b}=0
$$

The roots of $|M(\beta)|$ in (4) correspond to the allowed propagating hybrid modes in the PCF. The vector $b$ is then derived for each allowed mode by solving the Eigen-value problem in (4). The number of the very distinct small Eigen-values corresponds to the number of degenerate modes. This vector formulation was applied to the structure shown in Fig. 1. Some resulting modes are presented in Fig. 2

1. R. Ghosh et al, "Modal characteristics of fewmode silica-based photonic crystal fibers," Opt. and Quant. Elect. 32, 963-970 (2000).

2. Min Qiu, "Analysis of guided modes in Pho-
Fundamental mode(doublet)

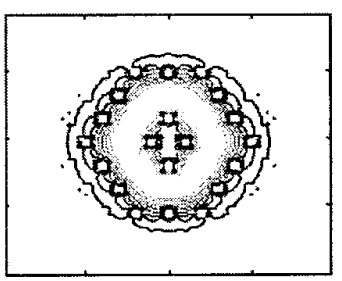

$E_{x}$ and $H_{y}$

First degenerate mode

$E_{y}$ and $H_{x}$

Second degeneratemode

Second order mode

(Four deg. modes)

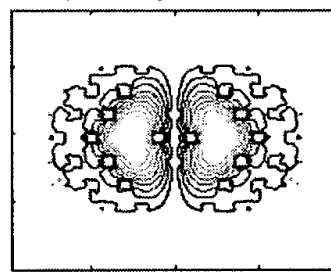

$E_{x}$ and $H_{y}$

First degenerate mode

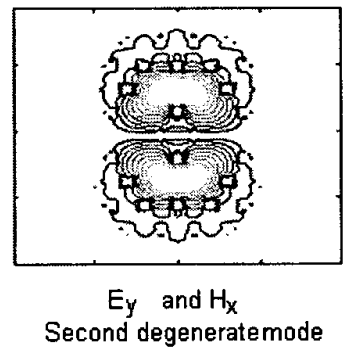

CMC5 Fig. 2. Fundamental and higher order modes for a ring cavity PCF. 